\title{
Species diversity and prey items of amphibians in Yoddom Wildlife Sanctuary, northeastern Thailand
}

\author{
PRAPAIPORN THONGPROH ${ }^{1, \nu}$, PRATEEP DUENGKAE ${ }^{2, v \varphi}$, PRAMOTE RATREE $^{3, v \vee v,}$ \\ EKACHAI PHETCHARAT T, ${ }^{4, v v v, ~ W A S S A N A ~ K I N G W O N G S A ~}{ }^{5, v v v v, ~ W E E Y A W A T ~ J A I T R O N G, ~}{ }^{6, v v v v v v, ~}$ \\ YODCHAIY CHUAYNKERN ${ }^{1, v v v v v v y}$, CHANTIP CHUAYNKERN ${ }^{1, v v v v v v v}$ \\ ${ }^{1}$ Department of Biology, Faculty of Science, Khon Kaen University, Mueang Khon Kaen, Khon Kaen, 40002, Thailand. Tel.: +6643-202531,

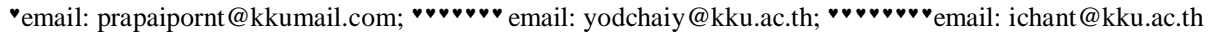 \\ ${ }^{2}$ Special Research Unit for Wildlife Genomics (SRUWG), Department of Forest Biology, Faculty of Forestry, Kasetsart University, Bangkok 10900, \\ Thailand."vemail: prateep.du@ku.ac.th \\ ${ }^{3}$ Protected Areas Regional Office 9 Ubon Ratchathani, Mueang Ubon Ratchathani, Ubon Ratchathani, 34000, Thailand. $v v^{\prime}$ email: ratree54@ hotmail.com \\ ${ }^{4}$ Royal Initiative Project for Developing Security in the Area of Dong Na Tam Forest, Sri Mueang Mai, Ubon Ratchathani, 34250, Thailand. \\ •vvemail:kofaku@gmail.com \\ ${ }^{5}$ Center of Study Natural and Wildlife, Nam Yuen, Ubon Ratchathani, 34260, Thailand. •vvvemail: kingwassana@gmail.com \\ ${ }^{6}$ Thailand Natural History Museum, National Science Museum, Technopolis, Khlong 5, Khlong Luang, Pathum Thani, 12120, Thailand,

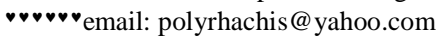

Manuscript received: 25 July 2019. Revision accepted: 28 August 2019.

\begin{abstract}
Thongproh P, Duengkae P, Ratree P, Phetcharat E, Kingwongsa W, Jaitrong W, Chuaynkern Y, Chuaynkern C. 2019. Species diversity and prey items of amphibians in Yoddom Wildlife Sanctuary, northeastern Thailand. Biodiversitas 20: $2718-2732$. Amphibian occurrence within Yoddom Wildlife Sanctuary, which is located along the border region among Thailand, Cambodia, and Laos, is poorly understood. To determine amphibian diversity within the sanctuary, we conducted daytime and nocturnal surveys from 2014 to 2017 within six management units. We recorded 26 amphibian species and two additional unidentified taxa from two orders (Anura and Gymnophiona) and six families. Four of the encountered species are new records for Ubon Ratchathani, (Kalophrynus interlineatus, Microhyla berdmorei, Micryletta inornata, and Chiromantis nongkhorensis). In addition to observational surveys, we investigated amphibian prey items via fecal pellet analyses. Prey items comprised mainly animals (85\% from class Insecta), but $9 \%$ of species consumed both plants and animals. Among insects, Coleoptera and Hymenoptera were the most abundant prey $(35 \%$ and $31 \%$, respectively). In addition, we observed one case of Ichthyophis kohtaoensis consuming an earthworm and an incident of cannibalism in Sylvirana mortenseni. Finally, due to illegal logging activities and active landmines, surveys were limited to the secure regions within the sanctuary. Thus, we noted that further species might remain unidentified within Yoddom Wildlife Sanctuary due to this limitation.
\end{abstract}

Keywords: Amphibian, diet, fecal pellet analysis, species richness

\section{INTRODUCTION}

The study of biodiversity provides the foundational data required to understand our natural resources at a global scale. Biodiversity data are not only necessary and valuable for conservation but for all fields of science (National Research Council 1992). Global amphibian species diversity knowledge is increasing rapidly $(8,043$ species are compiled in Frost 2019), yet the populations of these species are declining dramatically throughout the world (Whittaker et al. 2013; Heatwole and Das 2014). Major threats to amphibians, and to many other taxa, include habitat destruction, over-exploitation, alien species, chemical pollution, climate change, and infectious diseases, as well as additional sources of mortality such as road-kill, ultraviolet light, tourism, and anthropogenic-induced disasters (Hayes et al. 2010). Given these wide-ranging stressors, it is vital to increase survey efforts for amphibian fauna to provide data for global and local biodiversity assessments.

Thailand is experiencing a decline in amphibian populations, as are many other countries (Chuaynkern and Duengkae 2014). Thailand is central within the Oriental
Region, lying at the zoogeographical crossroads of Southeast Asia. Continental Thailand supports a fauna and flora with clear affinities to Myanmar and India to the west, Indo-China to the east, and mixed affinities to the north. In peninsular Thailand, the biota is predominantly Sundaic species, with an affinity to Malaysia and the more westerly islands of Indonesia (Hughes et al. 2003). Recorded amphibians in Thailand include approximately 175 species (Duengkae et al. 2016; Phusaensri et al. 2018; Thongproh et al. 2018; Frost 2019; Phimmachak et al. 2019) from three orders, Anura, Caudata, and Gymnophiona. Amphibian inventory efforts have been continuous in Thailand, but focused mainly on the discovery of new taxa (e.g., Phusaensri et al. 2018; Sheridan and Stuart 2018; Phimmachak et al. 2019). Inventory data from national parks, wildlife sanctuaries, and non-hunting areas, which represent major protected areas in the country, are published infrequently. Here, we report the results of an amphibian inventory and prey analysis from Yoddom Wildlife Sanctuary, Ubon Ratchathani, Thailand, and provide survey data for an area that was previously lacking such data. 


\section{MATERIALS AND METHODS}

\section{Study area}

Yoddom Wildlife Sanctuary (Figure 1; Department of National Parks, Wildlife and Plant Conservation 2006) is located in the lower portion of northeastern Thailand in Dom Pradit Subdistrict of the Nam Yuen District, in Ubon Ratchathani. Neighboring parks include Khao Phra Wihan National Park, Khao Phanom Dong Rak Wildlife Sanctuary, Huay Sala Wildlife Sanctuary, and Huay Tub Tun-Huay Samran Wildlife Sanctuary to the north; Phu Jong-Na Yoi National Park, Bun Ta Rik Yod Mon Wildlife Sanctuary, and an extensive forested area of southern Laos to the east; and forested areas of Cambodia and southern Laos to the west and south. The sanctuary is a part of the
Phanom Dong Rak mountain range, which runs along the border between Thailand and Cambodia.

Yoddom Wildlife Sanctuary covers $225.35 \mathrm{~km}^{2}$, and elevation ranges from 180 to 776 meters. The sanctuary is comprised of a headquarter area and five protected management units: Huay Chan Daeng, Wang Chao, Wang Yhai, Hauy Pra Chao, and Thum Nam Thip (Figure 1).

Currently, illegal logging takes place in the sanctuary, often targeting Siamese rosewood (Dalbergia cochinchinensis) (Department of National Parks, Wildlife and Plant Conservation 2012). In addition, the sanctuary contains active landmines. Due to these factors, field surveys can only be conducted in specific areas determined to be safe for research activities.

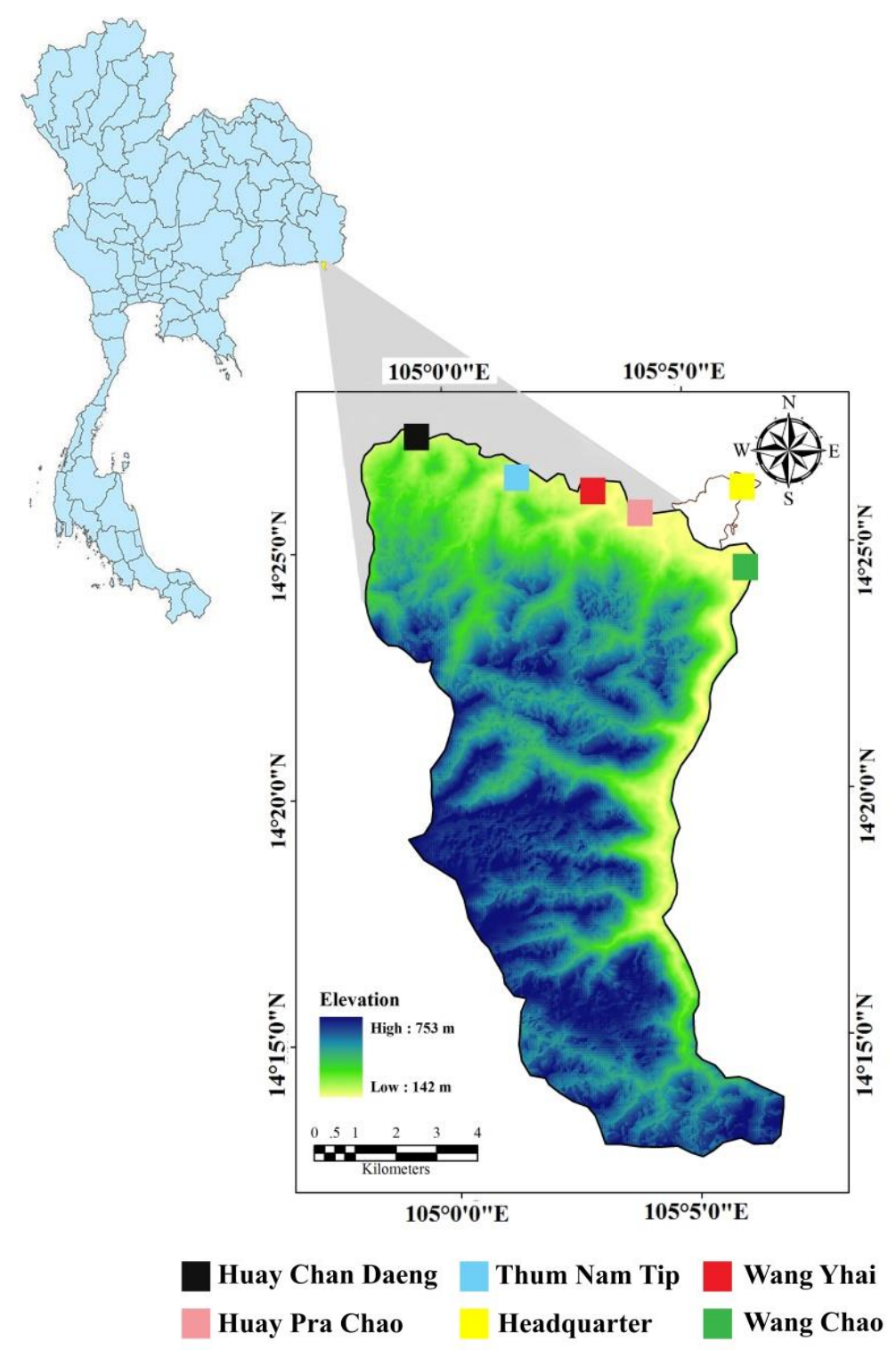

Figure 1. Map of Thailand showing the location of Yoddom Wildlife Sanctuary in the province of Ubon Ratchathani. The six protected units (the sanctuary, Huay Chan Daeng, Thum Nam Tip, Wang Yhai, Huay Pra Chao, the headquarter area, and Wang Chao) are shown in the inset panel. 
Table 1. List of specimens in this study that were collected from Yoddom Wildlife Sanctuary, Ubon Ratchathani Province, northeastern Thailand

\begin{tabular}{|c|c|c|}
\hline Taxon & $\begin{array}{l}\text { Number of } \\
\text { specimens }\end{array}$ & Collection number \\
\hline \multicolumn{3}{|l|}{ Bufonidae } \\
\hline Duttaphrynus melanostictus & 6 & $1252,1264,1589,1599-1600,2039$ \\
\hline \multicolumn{3}{|l|}{ Dicroglossidae } \\
\hline Occidozyga lima & 12 & $1254-1259,1574-1575,1797-1798,1971,1973$ \\
\hline Occidozyga martensii & 31 & $\begin{array}{l}1232,1234-1236,1281,1576-1582,1602,1605,1610-1611,1673-1674,1729,1734- \\
1736,1767,1769,1783,1901,1929,1996,1998,2034,2038\end{array}$ \\
\hline Fejervarya limnocharis & 11 & $1250,1270,1655,1671-1672,1711,1722,1910,1928,1977,2035-2036$ \\
\hline Fejervarya triora & 25 & $1276-1278,1658-1663,1738,1819,1885-1886,1911,1914,1917-1921,1923-1926,1990$ \\
\hline Hoplobatrachus rugulosus & 11 & $1253,1272,1292$, YC1291, 1877-1878, 1882 ( 5 tadpoles) \\
\hline Limnonectes lauhachindai & 8 & $1639,1733,1758,1913,1915-1916,1922,1927$ \\
\hline Limnonectes cf. savan & 11 & $1233,1263,1266,1271,1743,1745,1751,1753,1804,1818,2037$ \\
\hline \multicolumn{3}{|r|}{ 12JJ, 120J, 1200, 1271, 17 } \\
\hline Kaloula pulchra & 6 & $1231,1595,1560-1561,1737,1750$ \\
\hline Kaloula mediolineata & 4 & $1562,1596,1669,1723$ \\
\hline Microhyla berdmorei & 2 & 1268,1884 \\
\hline Microhyla butleri & 53 & $1201,1226-1227,1237,1606-1609,1688$, YC1284, 1970, 1981, 2032, 2075 (40 tadpoles) \\
\hline Microlyla heymonsi & 46 & $1219-1223,1267,1583-1586,1594,1668,1740,1770,1780,2076$ ( 31 tadpoles $)$ \\
\hline Microlyla fissipes & 17 & $\begin{array}{l}1228-1230,1238,1249,1274,1279-1280,1587-1588,1598,1601,1603-1604,1665,1732 \text {, } \\
2033\end{array}$ \\
\hline Microlyla pulchra & 10 & $1667,1983-1984,1991-1995,2000,2031$ \\
\hline Micryletta inornata & 8 & $1225,1566-1569,1744,1972,1982$ \\
\hline Kalophrynus interlineatus & 3 & $1224,1899-1900$ \\
\hline Glyphoglossus guttulatus & 8 & $1640,1883,1893-1897,1976$ \\
\hline Glyphoglossus molossus & 16 & $1275,1628-1631,1692,1296,1702-1710$ \\
\hline \multicolumn{3}{|l|}{ Ranidae } \\
\hline Hylarana erythraea & 8 & $1251,1564-1565,1713-1714,1725,1730,1974$ \\
\hline Hylarana macrodactyla & 17 & $1202-1206,1211-1215,1570-1573,1715,1784,1999$ \\
\hline "Hylarana lateralis" & 27 & $1632-1635,1678-1687,1689-1690,1693-1699,1691,1700-1701,1785$ \\
\hline Sylvirana mortenseni & 23 & $\begin{array}{l}1265,1269,1563,1666,1712,1717,1721,1731,1739,1746,1756,1759,1765,1794- \\
1796,1805-1806,1978-1980, \text { YD } 3-4\end{array}$ \\
\hline Odorrana sp. & 3 & $01716,01757,01760$ \\
\hline \multicolumn{3}{|l|}{ Rhacophoridae } \\
\hline Polypedates leucomystax & 46 & $\begin{array}{l}\text { 1209, 1216-1218, } 1273,1590-1593,1670,1726,1763-1764,1909,1912,1975,2074 \text { (17 } \\
\text { tadpoles), } 2077 \text { (13 tadpoles) }\end{array}$ \\
\hline Rhacophorus jarujini & 62 & $1755,1807,1902-1908,1985-1989,1937$ (46 tadpoles), YD1-2 \\
\hline Chiromantis nongkhorensis & 14 & $\begin{array}{l}\text { 1879-1880, 1887-1888, KS 01285, KS 01288-KS 01290, PS 01286, PS 01400-PS 01403, } \\
\text { YC } 01287\end{array}$ \\
\hline \multicolumn{3}{|r|}{ 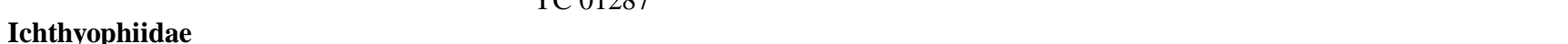 } \\
\hline Ichthyophis kohtaoensis & 2 & 1656,1793 \\
\hline
\end{tabular}

\section{Field survey and fecal pellet collecting}

We used a visual encounter survey technique (Heyer et al. 1994) for both daylight and nocturnal amphibian surveys in Yoddom Wildlife Sanctuary. Captured specimens were kept overnight in individual plastic bags for fecal pellet collection the following morning (Chuaynkern et al. 2009, Figure 2). All fecal pellets were preserved in $70 \%$ alcohol for further food-item analysis. For vouchering, amphibians were euthanized using chloretone and tagged with a unique number (Table 1). Then, specimens were injected using $10 \%$ formalin for fixative. Finally, specimens were transferred to $70 \%$ alcohol for preservation. For tadpole surveys, we searched breeding habitats such as rivers, ponds, tree holes, and ephemeral pools. Selected tadpoles were preserved in a 1:1 solution of $70 \%$ ethanol and $10 \%$ formalin (Inthara et al. 2009).
All specimens were cataloged and deposited at the Khon Kaen University Vertebrate Collection in Khon Kaen Province (Table 1). Research and specimen collection was performed with permission from the Department of National Parks, Wildlife and Plant Conservation (permit numbers 0907.4/2290 and 0097.4/11153).

\section{Prey item analysis}

Fecal pellet contents were identified using a stereomicroscope at the Faculty of Science at Khon Kaen University, Khon Kaen Province. Prey fragments found in pellets were identified via comparison with relevant taxonomic reference material for insects and taxa (White 1983; Triplehorn and Johnson 2005; Ponpituk et al. 2014; Ponpituk et al. 2015). Prey fragments were further photographed under the microscope. 


\section{RESULTS AND DISCUSSION}

\section{Species diversity}

We registered 28 amphibian species (27 frogs and one caecilian; $14.6 \%$ from Thailand richness, $0.34 \%$ from global richness) from 17 genera $(27.9 \%$ Thailand, $3.1 \%$ global), 6 families (60\% Thailand and $8 \%$ global) and 2 orders $(66.7 \%$ of Thailand and global) (Frost 2019) in Yoddom Wildlife Sanctuary (Table 2). The family Microhylidae contained the greatest diversity with 11 species. Of the recorded species, 22 are classified as Least Concern, two as Near Threatened, and one as Data Deficient by the International Union for Conservation of Nature (IUCN) Red List of Threatened Species (IUCN 2019). Under national conservation status ranks, 22 species are classified as Least Concern and three as Near Threatened, as evaluated by Nabhitabhata and Chan-ard (2005). No species was listed as a Protected Animal under the Wild Animal Reservation and Protection Act B.E. 2535 (1992).

Also, we provide here an updated checklist of amphibians for Yoddom Wildlife Sanctuary, based on our surveys and existing literature:

Class Amphibia Blainvill 1816

Order Anura Fischer von Waldheim 1813

Family Bufonidae Gray 1825

1. Duttaphrynus melanostictus (Schneider 1799)

Family Dicroglossidae Anderson 1817

2. Fejervarya limnocharis (Gravenhorst 1829)

3. Fejervarya triora Stuart, Chuaynkern, Chan-ard and Inger 2006

3. Hoplobatrachus rugulosus (Wiegmann 1834)

4. Limnonectes lauhachindai Aowphol, Rujirawan,

Taksinum, Chuaynkern and Stuart 2015

5. Limnonectes $\mathrm{cf}$. savan

6. Occidozyga lima (Gravenhorst 1829)

7. Occidozyga martensii (Peters 1867)

Family Microhylidae Günther 1858 (1843)

8. Glyphoglossus guttulatus (Blyth 1856)

9. Glyphoglossus molossus Günther 1869

10. Kalophrynus interlineatus (Blyth 1855)

11. Kaloula mediolineata Smith 1917

12. Kaloula pulchra Gray 1831

13. Microhyla berdmorei (Blyth 1856)

14. Microhyla butleri Boulenger 1900

15. Microhyla fissipes Boulenger 1884

16. Microhyla heymonsi Vogt 1911

17. Microhyla pulchra (Hallowell 1861)

18. Micryletta inornata (Boulenger 1890)

Family Ranidae Rafinesque 1814

19. Hylarana erythraea (Schlegel 1837)

20. Hylarana macrodactyla Günther 1858

21. "Hylarana lateralis" (Boulenger 1887)

22. Sylvirana mortenseni (Boulenger 1903)

23. Odorrana sp.

Family Rhacophoridae Hoffman 1932 (1858)

25. Chiromantis nongkhorensis (Cochran 1927)

26. Polypedates leucomystax (Gravenhorst 1829)

27. Rhacophorus jarujini Matsui and Panha 2006
Order Gymnophiona Müller 1832

Family Ichthyophiidae Taylor 1968

28. Ichthyophis kohtaoensis Taylor 1960

\section{Prey items}

We collected 70 fecal pellets from 25 species of the 28 registered species (Table 3). Fecal pellets were not obtained from Odorrana sp., Microhyla berdmorei, or Ichthyophis kohtaoensis. Analysis of prey found in fecal pellets indicated that the majority of species consumed mainly animal material, with only a few species consuming both animal and plant material (Hylarana erythraea, Limnonectes lauhachindai, Occidozyga lima, Occidozyga martensii, and Rhacophorus jarujini). The most prevalent animal prey belonged to the classes Coleoptera and Hymenoptera. Photographed encounters during field surveys documented an earthworm consumed by Ichthyophis kohtaoensis and a juvenile frog consumed by Sylvirana mortenseni.

\section{Prey items and species diversity}

In our amphibian surveys, we registered 28 amphibians in Yoddom Wildlife Sanctuary (Table 2). Among these, four species were new provincial records for Ubon Ratchathani: Kalophrynus interlineatus, Microhyla berdmorei, Micryletta inornata, and Chiromantis nongkhorensis. Two taxa (Limnonectes cf. savan and Odorrana sp.) require further detailed study or an alternative identification technique aside from external morphological comparisons. In Thailand, four species of the genus Sylvirana have been reported (Sylvirana faber, Sylvirana malayana, Sylvirana mortenseni, and Sylvirana nigrovittata), and all have a very similar morphology (Sheridan and Stuart 2018). Sylvirana nigrovittata and Sylvirana mortenseni were previously reported from Ubon Ratchathani (Sheridan and Stuart 2018), but their highly similar external morphology makes them difficult to distinguish. Sheridan and Stuart (2018) reported that Sylvirana mortenseni is significantly larger than Sylvirana nigrovittata. However, the measurements of 12 characters on these species showed overlap between adult males and females of both species (Sheridan and Stuart 2018). We, therefore, assigned our specimens to Sylvirana mortenseni, because the collection site was in close proximity to the location of a Sylvirana mortenseni observation in Sheridan and Stuart (2018)

We did not encounter three species that were previously reported in the province: Occidozyga magnapustulosus, Limnonectes gyldenstolpei, and Indosylvirana milleti. Occidozyga magnapustulosus (type locality: "Ban Na Phua (subvillage), Kan Luang (village), $\mathrm{Na}$ Kae (district), Nakhon Phanom (province), Thailand, elevation approx. 200 m") was described in Nakhon Phanom by Taylor and Elbel (1958). This species is similar in morphology to Occidozyga martensii but different in that it bears large tubercles on the dorsum with surmounted tiny tubercles (Taylor 1962). Occidozyga magnapustulosus has been reported in several parts of Thailand including Chiang Mai, Nakhon Phanom, Loei, Sakon Nakhon, and Ubon 
Ratchathani (Chuaynkern and Chuaynkern 2012; Duengkae et al. 2016). Concerning Limnonectes gyldenstolpei (type locality: "Bang Hue Pong, a small village on the southern slopes of the Koon Tan Mountains, Northern Siam [Thailand]"), Ohler and Dubois (1999) demonstrated that Elachyglossa gyldenstolpei is a senior subjective synonym of Rana pileata. Therefore, the name Rana pileata was replaced by Elachyglossa gyldenstolpei, which has since been moved to the genus Limnonectes. Presently, Limnonectes gyldenstolpei has been identified in several areas throughout Thailand (Duengkae et al. 2016). Indosylvirana milleti is a species that is very similar in morphology to Sylvirana nigrovittata but is distinguished by its smaller overall size, smaller head, forearm, and webbing and a larger tympanum (see Chuaynkern et al. 2004). This species was reported in the provinces of Chanthaburi, Chachoengsao, Trat, Nakhon Nayok, Nakhon
Ratchasima, Ubon Ratchathani, and Amnat Charoen (Chuaynkern et al. 2004; Chuaynkern 2005; Duengkae et al. 2016.). However, the reported occurrence of Indosylvirana milleti in Ubon Ratchathani was based on the literature (Chan-ard 2003), and no voucher specimen exists. It is possible that these three species occur in Yoddom Wildlife Sanctuary, but we were unable to encounter them due to the safety constraints on our survey area, which was limited by illegal logging and landmines. Also, it is possible that additional species and populations occur in the forested areas of the sanctuary, along the borders of Laos and Cambodia. Despite these safety concerns, it is vital to increase the inventory of amphibians in this area given that we are facing a global biodiversity crisis (Leakey and Lewin 1996; May 2002), and populations of species are declining worldwide (Stuart et al. 2008).

Table 2. List of amphibian species recorded within Yoddom Wildlife Sanctuary and their associated conservation status. Nomenclature follows Frost (2019). IUCN = International Union for Conservation of Nature and Natural Resources (www.iucnredlist.org), ONEP = Office of Natural Resources and Environmental Policy and Planning (2017). TA = tadpoles, AD = adults, DD = Data Deficient, LC = Least Concern, and NT = Near Threatened.

\begin{tabular}{|c|c|c|c|c|}
\hline \multirow{2}{*}{ Taxa } & \multicolumn{2}{|c|}{ Specimens } & \multicolumn{2}{|c|}{ Conservation Status } \\
\hline & TA & AD & IUCN & ONEP \\
\hline \multicolumn{5}{|l|}{ Class Amphibia Blainvill 1816} \\
\hline \multicolumn{5}{|l|}{ Order Anura Fischer von Waldheim 1813} \\
\hline \multicolumn{5}{|l|}{ Family Bufonidae Gray 1825} \\
\hline Duttaphrynus melanostictus (Schneider 1799) & & $\mathrm{X}$ & $\mathrm{LC}$ & $\mathrm{LC}$ \\
\hline \multicolumn{5}{|l|}{ Family Dicroglossidae Anderson 1817} \\
\hline Fejervarya limnocharis (Gravenhorst 1829) & & $\mathrm{X}$ & $\mathrm{LC}$ & $\mathrm{LC}$ \\
\hline Fejervarya triora Stuart, Chuaynkern, Chan-ard and Inger 2006 & & $\mathrm{X}$ & $\mathrm{LC}$ & $\mathrm{LC}$ \\
\hline Hoplobatrachus rugulosus (Wiegmann 1834) & $\mathrm{X}$ & $\mathrm{X}$ & $\mathrm{LC}$ & $\mathrm{LC}$ \\
\hline Limnonectes lauhachindai Aowphol, Rujirawan, Taksinum, Chuaynkern and Stuart 2015 & & $\mathrm{X}$ & - & - \\
\hline Limnonectes cf. savan & & $\mathrm{X}$ & - & - \\
\hline Occidozyga lima (Gravenhorst 1829) & & $\mathrm{X}$ & $\mathrm{LC}$ & $\mathrm{LC}$ \\
\hline Occidozyga martensii (Peters 1867) & & $\mathrm{X}$ & $\mathrm{LC}$ & $\mathrm{LC}$ \\
\hline \multicolumn{5}{|l|}{ Family Microhylidae Günther 1858 (1843) } \\
\hline Glyphoglossus guttulatus(Blyth 1856) & & $\mathrm{X}$ & $\mathrm{LC}$ & $\mathrm{LC}$ \\
\hline Glyphoglossus molossus Günther 1868 & & $\mathrm{X}$ & NT & NT \\
\hline Kalophrynus interlineatus (Blyth 1855) & & $X$ & $\mathrm{LC}$ & $\mathrm{LC}$ \\
\hline Kaloula mediolineata Smith 1917 & & $\mathrm{X}$ & NT & NT \\
\hline Kaloula pulchra Gray 1831 & & $\mathrm{X}$ & $\mathrm{LC}$ & $\mathrm{LC}$ \\
\hline Microhyla berdmorei (Blyth 1856) & & $\mathrm{X}$ & $\mathrm{LC}$ & $\mathrm{LC}$ \\
\hline Microhyla butleri Boulenger 1900 & $\mathrm{X}$ & $\mathrm{X}$ & $\mathrm{LC}$ & $\mathrm{LC}$ \\
\hline Microhyla fissipes Boulenger 1884 & & $\mathrm{X}$ & $\mathrm{LC}$ & $\mathrm{LC}$ \\
\hline Microhyla heymonsi Vogt 1911 & $\mathrm{X}$ & $\mathrm{X}$ & $\mathrm{LC}$ & $\mathrm{LC}$ \\
\hline Microhyla pulchra (Hallowell 1861) & & $\mathrm{X}$ & $\mathrm{LC}$ & $\mathrm{LC}$ \\
\hline Micryletta inornata (Boulenger 1890) & & $\mathrm{X}$ & $\mathrm{LC}$ & $\mathrm{LC}$ \\
\hline \multicolumn{5}{|l|}{ Family Ranidae Rafinesque $\mathbf{1 8 1 4}$} \\
\hline "Hylarana lateralis" (Boulenger 1887) & & $X$ & $\mathrm{LC}$ & NT \\
\hline Hylarana erythraea (Schlegel 1837) & & $\mathrm{X}$ & $\mathrm{LC}$ & $\mathrm{LC}$ \\
\hline Hylarana macrodactyla Günther 1858 & & $\mathrm{X}$ & $\mathrm{LC}$ & $\mathrm{LC}$ \\
\hline Sylvirana mortenseni (Boulenger 1903) & & $\mathrm{X}$ & $\mathrm{LC}$ & $\mathrm{LC}$ \\
\hline Odorrana sp. & & $\mathrm{X}$ & - & - \\
\hline \multicolumn{5}{|l|}{ Family Rhacophoridae Hoffman 1932 (1858) } \\
\hline Chiromantis nongkhorensis (Cochran 1927) & & $\mathrm{X}$ & $\mathrm{LC}$ & $\mathrm{LC}$ \\
\hline Polypedates leucomystax (Gravenhorst 1829) & $\mathrm{X}$ & $\mathrm{X}$ & $\mathrm{LC}$ & $\mathrm{LC}$ \\
\hline Rhacophorus jarujini Matsui and Panha 2006 & $\mathrm{X}$ & $X$ & $\mathrm{DD}$ & $\mathrm{LC}$ \\
\hline \multicolumn{5}{|l|}{ Order Gymnophiona Müller 1832} \\
\hline \multicolumn{5}{|l|}{ Family Ichthyophiidae Taylor 1968} \\
\hline Ichthyophis kohtaoensis Taylor 1960 & & $\mathrm{X}$ & $\mathrm{LC}$ & $\mathrm{LC}$ \\
\hline
\end{tabular}



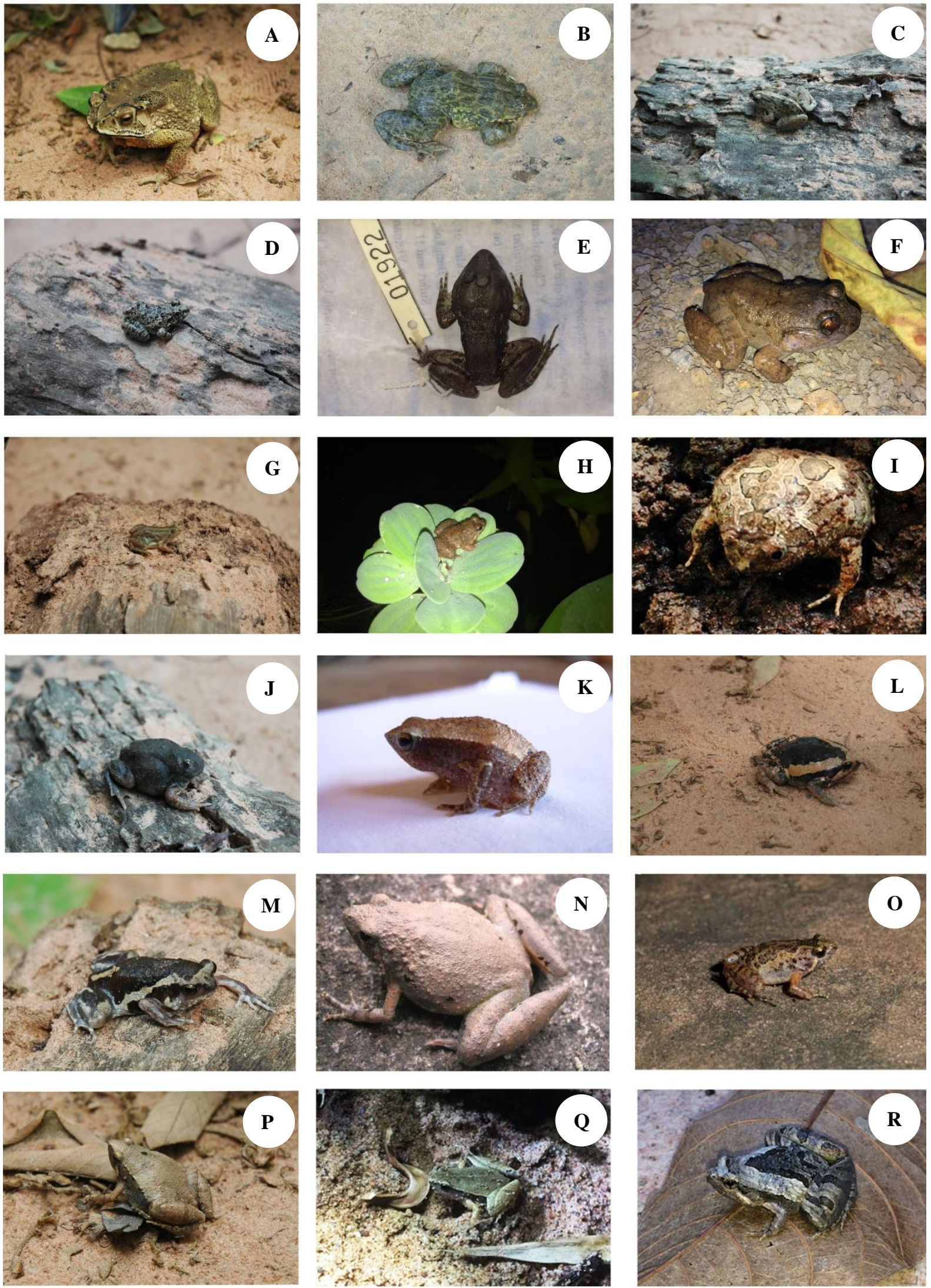

Figure 2. Amphibians registered in Yoddom Wildlife Sanctuary. A. Duttaphrynus melanostictus, B. Hoplobatrachus rugulosus, C. Fejervarya limnocharis, D. Fejervarya triora, E. Limnonectes lauhachindai, F. Limnonectes cf. savan, G. Occidozyga lima, H. Occidozyga martensii, I. Glyphoglossus guttulatus, J. Glyphoglossus molossus, K. Kalophrynus interlineatus, L. Kaloula pulchra, M. Kaloula mediolineata, N. Microhyla berdmorei, O. Microhyla butleri, P. Microhyla fissipes, Q. Microhyla heymonsi, R. Microhyla pulchra. 

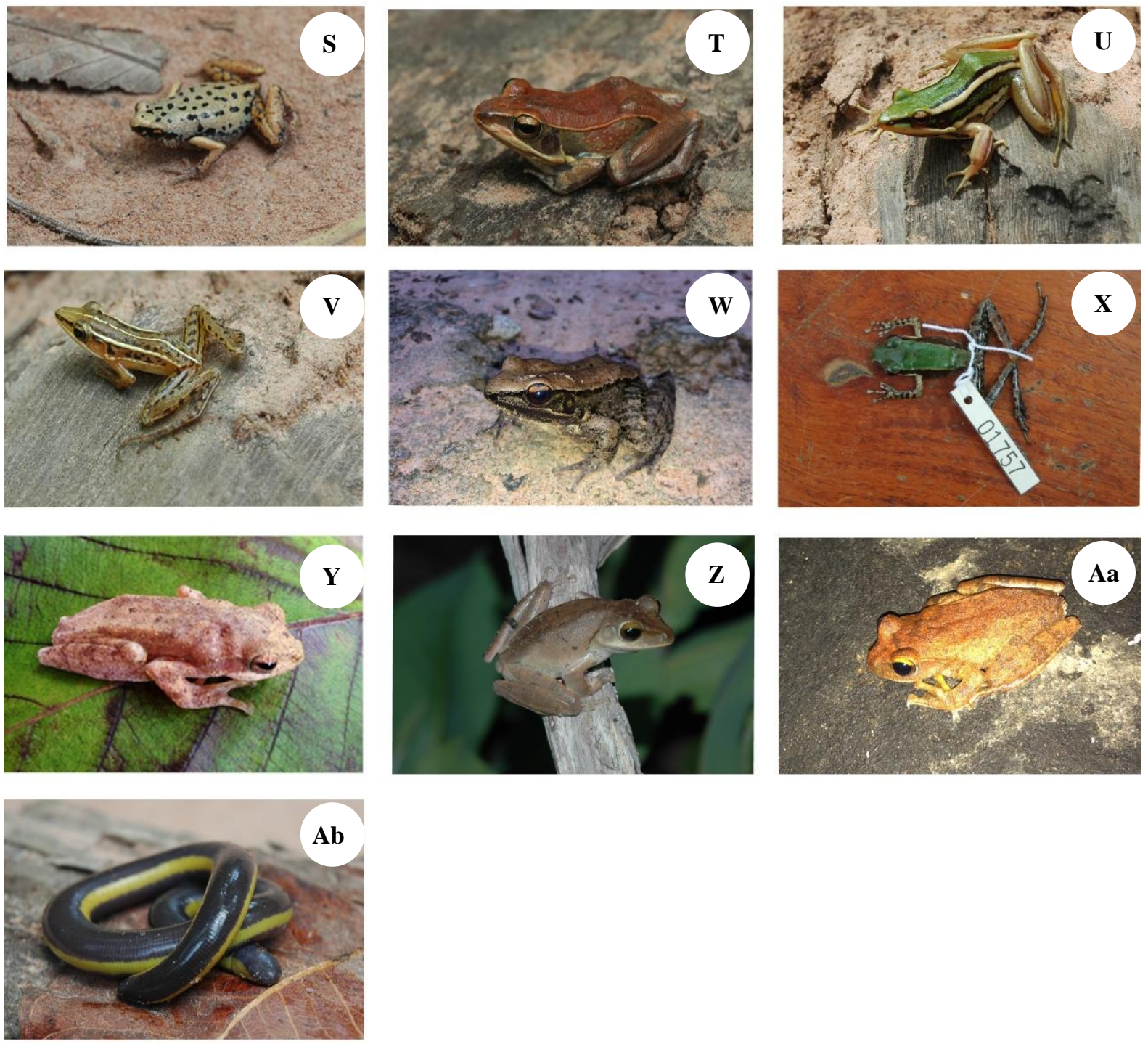

Figure 3. Amphibians registered in Yoddom Wildlife Sanctuary. S. Micryletta inornata, T. "Hylarana lateralis", U. Hylarana erythraea, V. Hylarana macrodactyla, W. Sylvirana mortenseni, X. Odorrana sp., Y. Chiromantis nongkhorensis, Z. Polypedates leucomystax, Aa. Rhacophorus jarujini, Ab. Ichthyophis kohtaoensis

Some amphibian species found in Yoddom Wildlife Sanctuary have been evaluated using global conservation status assessments. Whereas none were included in the list of protected animals according to the Wildlife Preservation and Protection Act, B.E. 2535 (1992), they are protected by law (The Government Gazette 2003). Collecting amphibians in protected area such as Yoddom Wildlife Sanctuary without permission from the Department of National Parks, Wildlife and Plant Conservation is illegal. However, many amphibians and their tadpoles are consumed as food by local people surrounding the sanctuary, including Fejervarya limnocharis, Fejervarya triora, Hoplobatrachus rugulosus, Limnonectes lauhachindai, Occidozyga lima, Occidozyga martensii, Glyphoglossus guttulatus, Glyphoglossus molossus, Kaloula mediolineata, Kaloula pulchra, Microhyla berdmorei, Microhyla butleri, Microhyla fissipes, Microhyla heymonsi, Microhyla pulchra, "Hylarana lateralis", and Sylvirana mortenseni (Duengkae et al. 2016). These species are consumed and/or sold in local markets, but only Hoplobatrachus rugulosus is used in aquaculture. Hunting for consumption is one of the major threats noted in amphibian decline (Stuart et al. 2008; Chuaynkern and Duengkae 2014). Thus, area management and law enforcement are critical challenges for government officials in the current biodiversity crisis. 


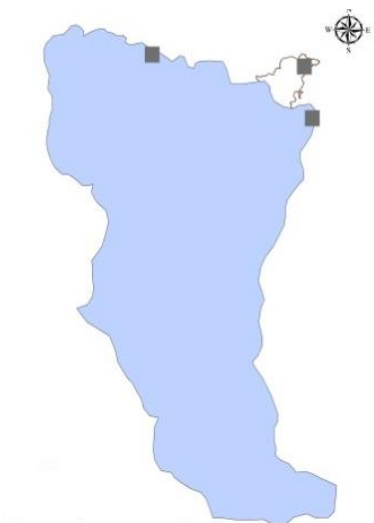

Duttaphrynus melanostictus

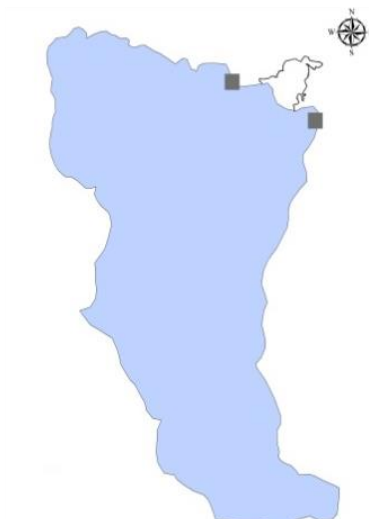

Limnonectes lauhachindai

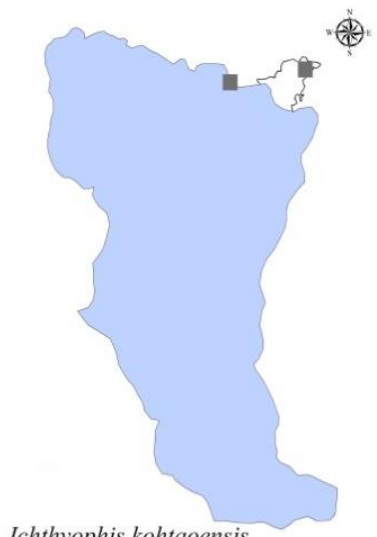

Ichthyophis kohtaoensis

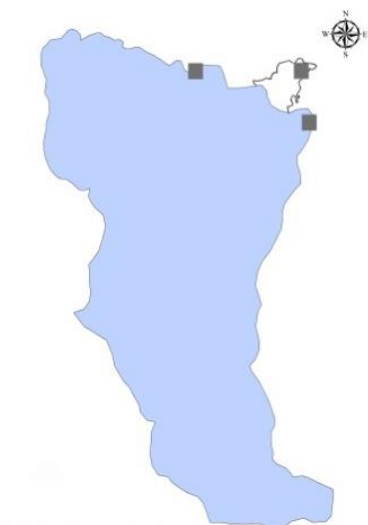

Kaloula mediolineata

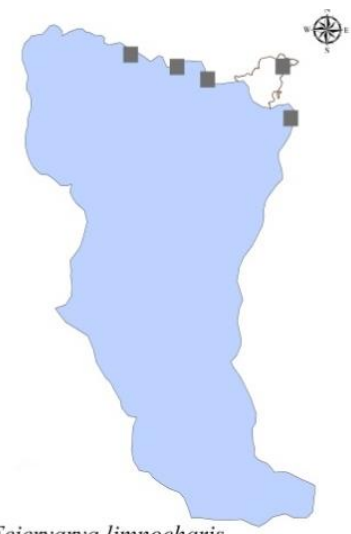

Fejervarya limnocharis

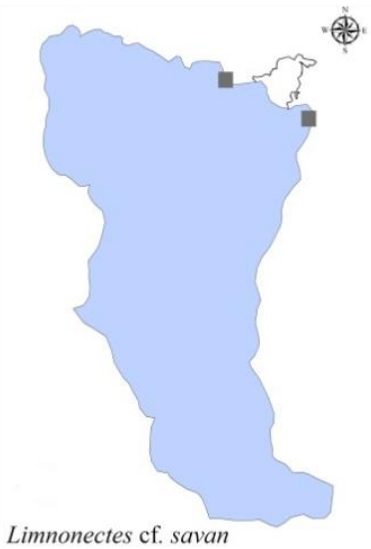

Limnonectes cf. savan
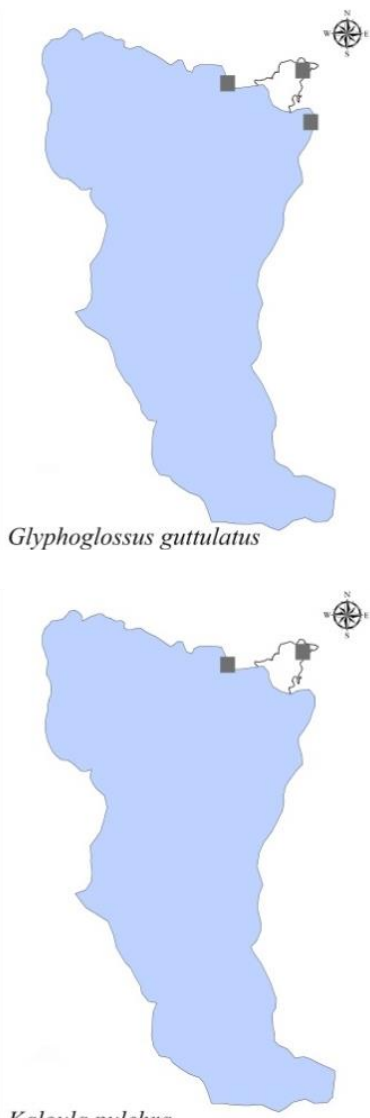

Kaloula pulchra

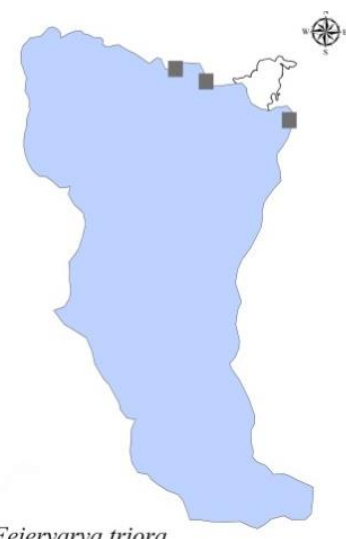

Fejervarya triora
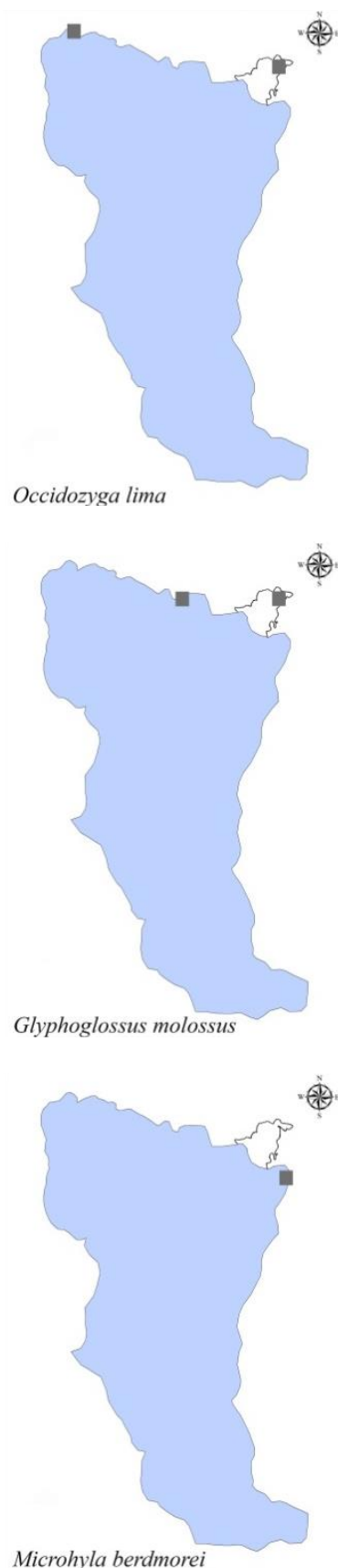

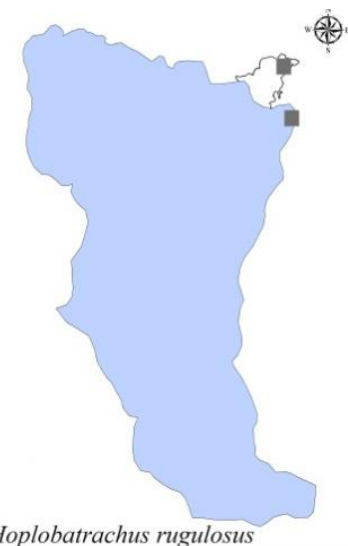

Hoplobatrachus rugulosus

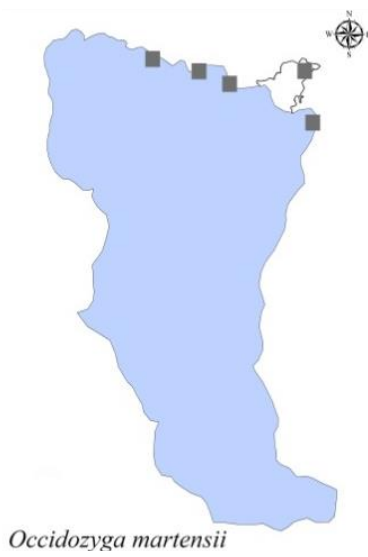

Occidozyga martens
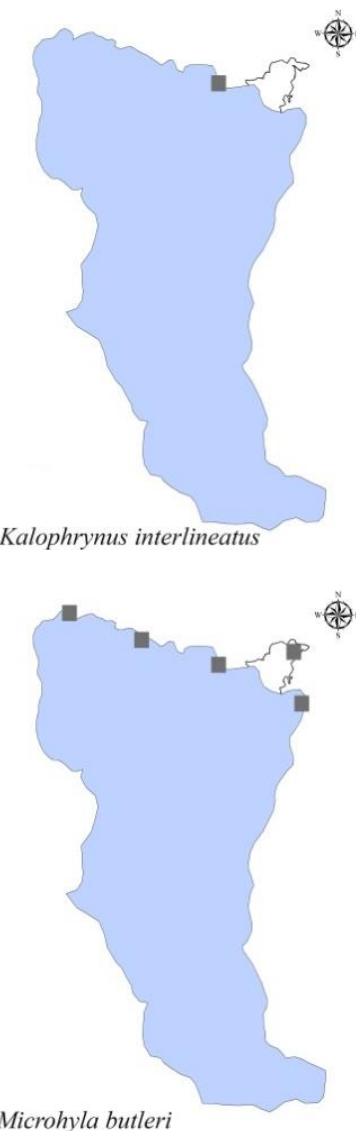

Figure 4. Management units in which amphibian species were observed in Yoddom Wildlife Sanctuary, Thailand 


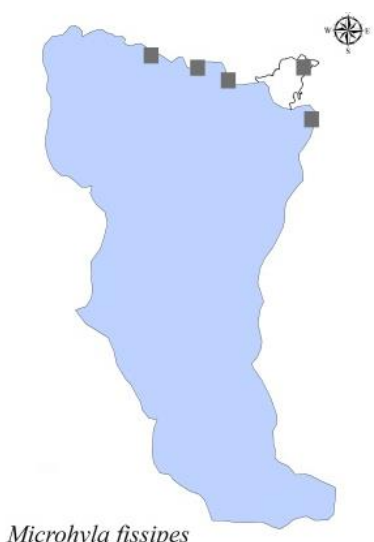

Microhyla fissipes

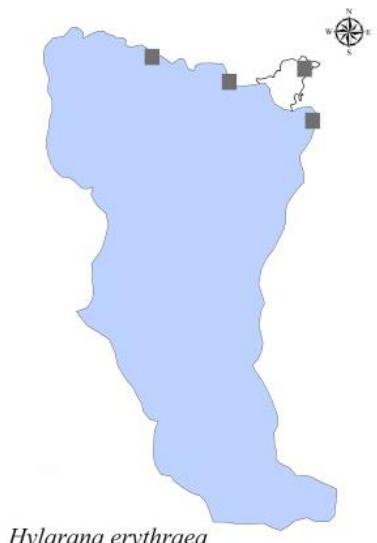

Hylarana erythraea

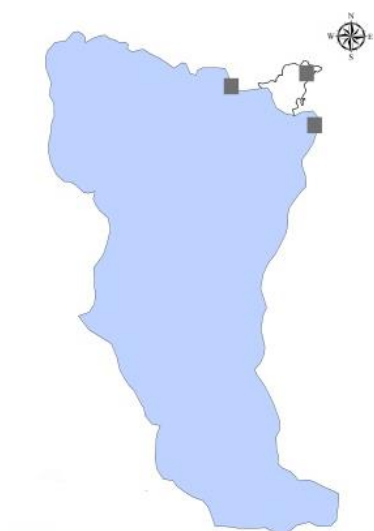

Sylvirana mortenseni

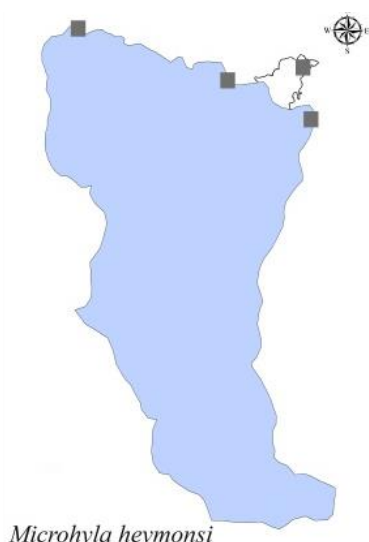

Microhyla heymonsi

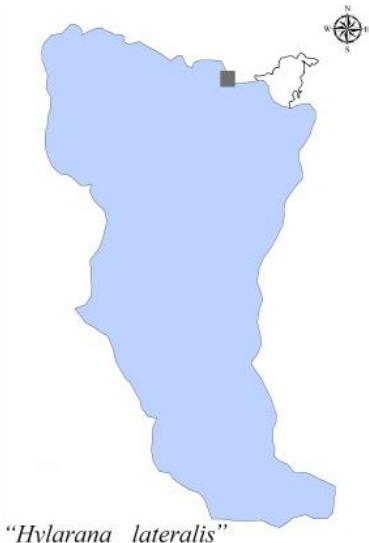

"Hylarana lateralis"

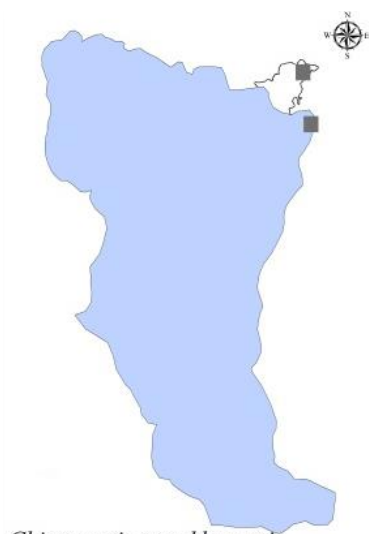

Chiromantis nongkhorensis

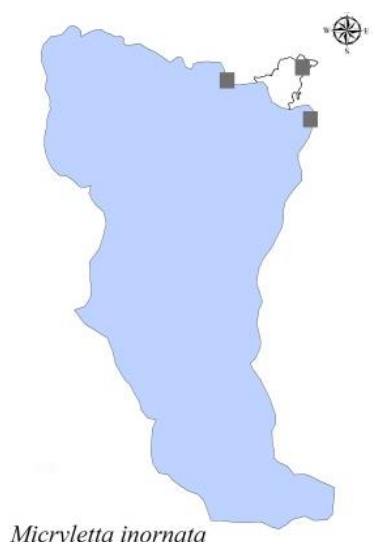

Micryletta inornata
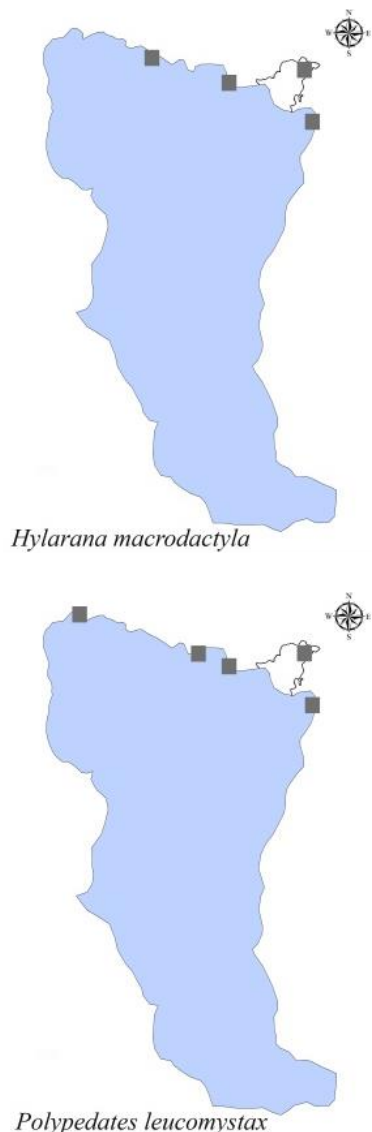

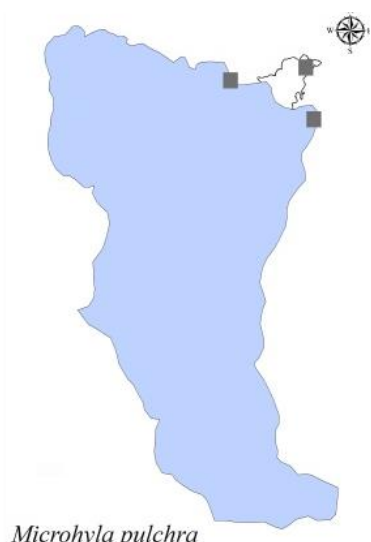

Microhyla pulchra

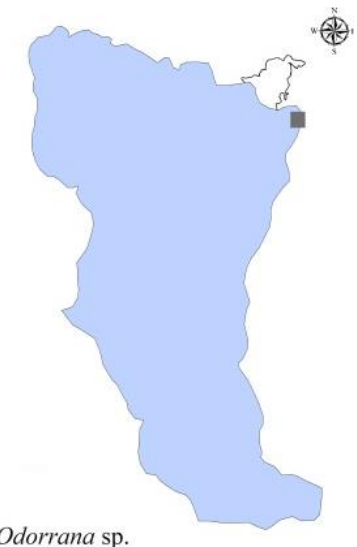

Odorrana sp.

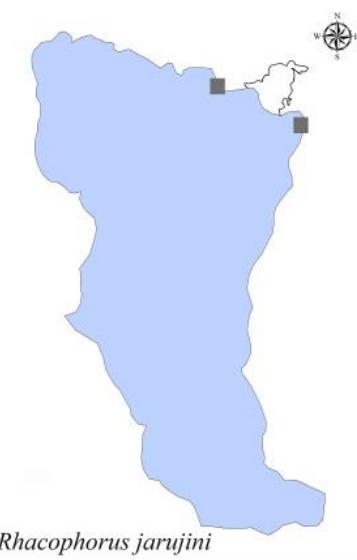

Figure 5. Management units in which amphibian species were observed in Yoddom Wildlife Sanctuary, Thailand (cont'd).
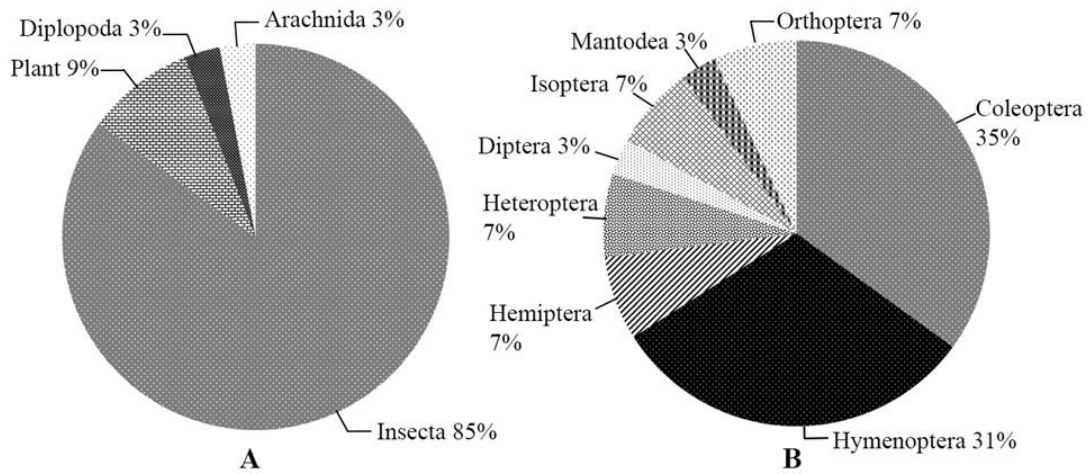

Figure 6. Percentage of various prey items found in amphibian fecal pellets from 25 species captured in Yoddom Wildlife Sanctuary, Thailand. A. Proportion of identified prey items belonging to each prey group, B. Proportion of prey items belonging to class Insecta. 
Table 3. Prey items found in fecal pellets from 25 amphibian species captured in Yoddom Wildlife Sanctuary, Thailand

\begin{tabular}{|c|c|c|c|c|c|c|c|c|c|c|c|c|c|c|c|c|c|c|c|c|c|c|c|c|c|c|}
\hline Kingdom & Phylum & Class & Order & Family & Species & 离 & 皇 & $\stackrel{\Xi}{E}$ & 管 & 売 & & & 苛 & 鹿 & 音 & 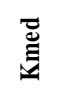 & & 志 & & 总 & 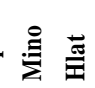 & 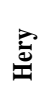 & 苋 & 苛 & & 荤 \\
\hline Plantae & Bryophyta & & & & & & & & & & + & + & & & & & & & & & & & & & & \\
\hline Plantae & Magnoliophyta & Magnoliopsida & & & & & & & $+t$ & + & & + & + & & & & & & & & & & & & & + \\
\hline Plantae & Magnoliophyta & Liliopsida & Poales & Poaceae & & & & & & & & & & & & & & & & & & & + & & & \\
\hline Animalia & Arthropoda & Arachnida & & & & & & & + & & & + & + & & & & & + & & & & & & & & \\
\hline Animalia & Arthropoda & Diplopoda & Sphaerotheriida & Zephroniidae & & + & & & & & & & & & & & & & & & & & & & & \\
\hline Animalia & Arthropoda & Insecta & Coleoptera & Cerambycidae & & & & & & & & & & & & & & & & & & & & & + & \\
\hline Animalia & Arthropoda & Insecta & Coleoptera & Chrysomelidae & & & & & & & + & & & & & & & & + & & & & & + & & \\
\hline Animalia & Arthropoda & Insecta & Coleoptera & Curculionidae & & + & + & & & & & & & & & & & & & & & + & & & & \\
\hline Animalia & Arthropoda & Insecta & Coleoptera & Elateridae & & & + & & & & + & & + & & & + & & + & & & & & & & & \\
\hline Animalia & Arthropoda & Insecta & Coleoptera & Lucanidae & & & & & & & & & & & & & & & & & & + & & & & \\
\hline Animalia & Arthropoda & Insecta & Coleoptera & Scarabaeidae & & & + & & & & & & & & & & & & & & + & + & & + & & \\
\hline Animalia & Arthropoda & Insecta & Coleoptera & Scolytidae & & & & & & & & & & & & & & + & & & & & & & & \\
\hline Animalia & Arthropoda & Insecta & Coleoptera & Tenebrionidae & & & + & & & & ++ & + & + & & & & & + & & & & + & & & & \\
\hline Animalia & Arthropoda & Insecta & Coleoptera & & & & & + & & + & & & & & & & & & & & & & + & & & + \\
\hline Animalia & Arthropoda & Insecta & Coleoptera (larva) & & & & & & & & & + & + & & & & & & & & & & & & & \\
\hline Animalia & Arthropoda & Insecta & Diptera & & & & + & & & & & & & & & & & & & & & + & & + & & \\
\hline Animalia & Arthropoda & Insecta & Hemiptera & Gerridae & & & + & & & & + & & & & & & & & & & & & & + & & \\
\hline Animalia & Arthropoda & Insecta & Hemiptera & & & & & & & & & & & & & & & & & & & + & + & & & \\
\hline Animalia & Arthropoda & Insecta & Heteroptera & Reduviidae & & & & & & & + & & & & & & & & & & & & & & & \\
\hline Animalia & Arthropoda & Insecta & Heteroptera & & & & + & & & & & & & & & & & & & & & & & & & \\
\hline Animalia & Arthropoda & Insecta & Hymenoptera & Formicidae & Calyptomyrmex rectopilosus & & & & & & & & & & & & & & & & + & & & & & \\
\hline Animalia & Arthropoda & Insecta & Hymenoptera & Formicidae & Camponotus sp. & & + & & & & + & + & & & & & & & ++ & + & + & & & & & \\
\hline Animalia & Arthropoda & Insecta & Hymenoptera & Formicidae & Carebara diversa & & & & & & & & & & & & & + & $+\quad+$ & + & + & & + & + & & \\
\hline Animalia & Arthropoda & Insecta & Hymenoptera & Formicidae & Crematogaster aurita & & & & & & & & & & & + & ++ & + & + & & & & & & & \\
\hline Animalia & Arthropoda & Insecta & Hymenoptera & Formicidae & Ectomomyrmex sp. & & & & & & & & & & & & & + & & & & & & & & \\
\hline Animalia & Arthropoda & Insecta & Hymenoptera & Formicidae & Leptogenys sp. & & & & & & & & & & + & & + & & & & & & & & & \\
\hline Animalia & Arthropoda & Insecta & Hymenoptera & Formicidae & Odontoponera denticulata & & + & + & & & & & + & & + & & ++ & + & ++ & ++ & & & + & + & & \\
\hline Animalia & Arthropoda & Insecta & Hylmenoptera & Formicidae & Pheidole sp. & & & & & & & + & + & & & + & & & & & & + & + & & & \\
\hline Animalia & Arthropoda & Insecta & Hylmenoptera & Formicidae & Tetramorium sp. & & & & & & & & & & & & & & + & + & & & & & & \\
\hline Animalia & Arthropoda & Insecta & Isoptera & Rhinotermitidae & & & & & & & & + & + & & & & & & & & & & & & & \\
\hline Animalia & Arthropoda & Insecta & Isoptera & Termitidae & & & + & & & & & & & + & & & & & ++ & & + & & & + & + & \\
\hline Animalia & Arthropoda & Insecta & Mantodea & Hymenopodidae & & & & & & & & + & + & & & & & & & & & & & & & \\
\hline Animalia & Arthropoda & Insecta & Orthoptera & Acrididae & & & & & + & & & & & & & & & & & & & & & + & & \\
\hline Animalia & Arthropoda & Insecta & Orthoptera & & & & + & + & & & & & & & & + & + & & + & & & + & + & + & + & \\
\hline
\end{tabular}

( $\mathrm{Kpul}=$ Kaloula pulchra, Mbut = Microhyla butleri, Mfis = Microhyla fissipes, Mhey = Microhyla heymonsi, Mpul = Microhyla pulchra, Mino $=$ Micryletta inornata, Hlat $="$ Hylarana lateralis", Hery = Hylarana erythraea, Hmac = Hylarana macrodactyla, Smor = Sylvirana mortenseni, Cnon = Chiromantis nongkhorensis, Pleu = Polypedates leucomystax, and Rjar = Rhacophorus jarujini. 

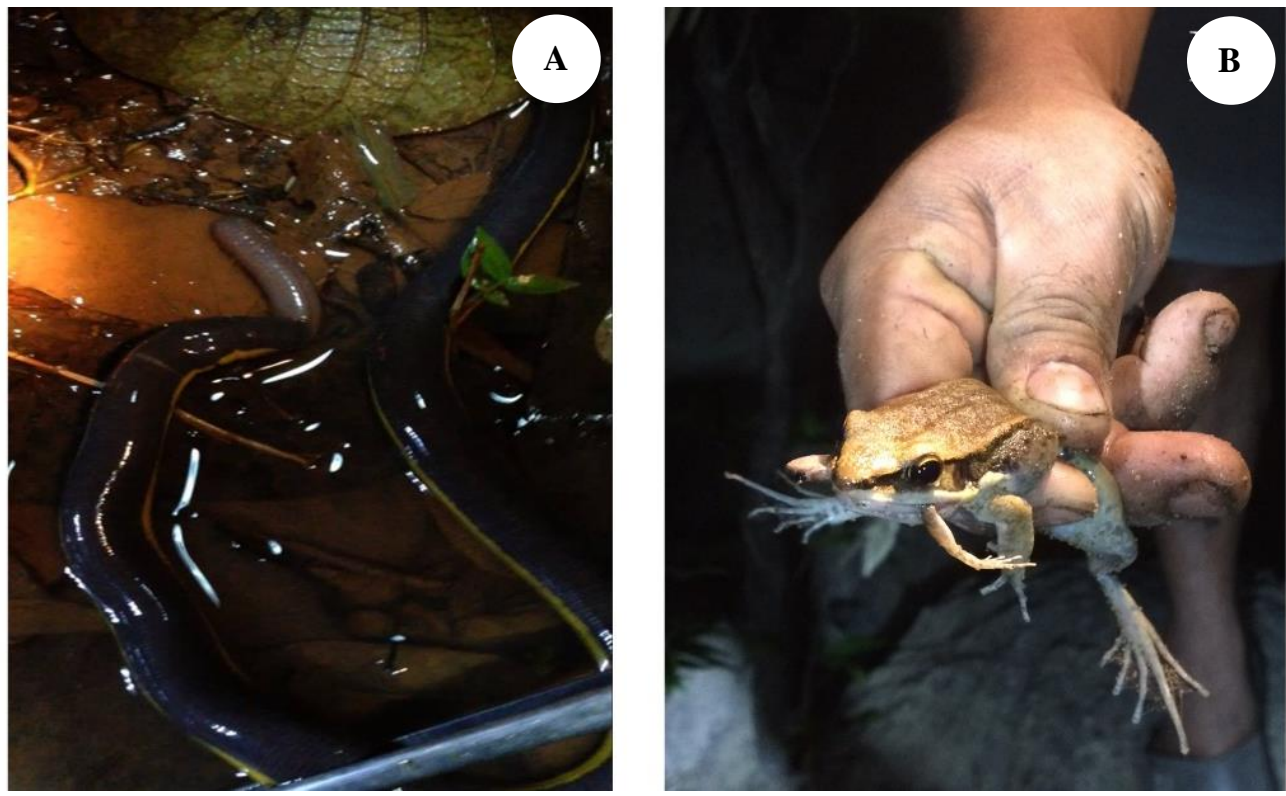

Figure 7. A. Ichthyophis kohtaoensis (Ichthyophiidae) swallowing an earthworm on a roadside in the headquarter management unit (photography by Nattapon Surachotmongkol), B. Sylvirana mortenseni (Ranidae), caught by hand while cannibalizing a juvenile Sylvirana mortenseni at Lam Dom Yai River in Wang Chao Protected Unit (photography by Prapaiporn Thongproh)

Prey item analysis showed that amphibians in the study area consumed mainly insects in the orders Coleoptera (35\%) and Hymenoptera (31\%). Identification of prey items indicated that amphibians in this study were generalist predators, rather than specialists. Prey in the family Formicidae were the most commonly consumed, which aligns with previous findings (Chuaynkern et al. 2009; Ponpituk et al. 2015). Foods items consumed by Duttaphrynus melanostictus (two pellets) contained only class Diplopoda (family Zephroniidae) and Insecta (family Curculionidae). However, Norval et al. (2014), in a sample of 77 stomach contents, noted much more diverse prey for this species (Arachnida, Chilopoda, Clitelata, Diplopoda, Gastropoda, and Insecta). Norval et al. (2014) further found that Fejervarya limnocharis consumed two classes (Arachnida and Insecta), while our work found only class Insecta in a smaller sample size (7 pellets vs. 10 stomach contents). Kalophrynus interlineatus (one pellet) consumed two taxa in the family Formicidae, which are similar findings to that of Chuaynkern et al. (2009), who reported six ant and beetle taxa. Microhyla fissipes (five pellets) consumed a variety of insects, including Coleoptera (family Chrysomelidae), Hymenoptera (Formicidae), and Isoptera (Termitidae) in our study, whereas Norval et al. (2014) reported that this species consumed mainly Insecta and Arachnida, based on 39 stomach contents. Microhyla heymonsi consumed two insect families (Formicidae and Termitidae, four pellets) in our study, whereas Norval at al. (2014) found Arachnida and Insecta in 20 stomach content samples.
We observed Ichthyophis kohtaoensis consuming an earthworm (Annelida). Kupfer and Maraun (2003) first reported oribatid mites (Oribatida) as the major prey of Ichthyophis kohtaoensis and further claimed (based on the personal observations of Alexander Kupfer) that the caecilian diet consists primarily of earthworms, termites, and ants. Ngo et al. (2014) found several groups of prey items in 135 stomach content samples of the related Ichthyophis bannanicus from the Mekong Delta in Vietnam, including Annelida (Lumbricidae [earthworms]), Arachnida (Araneae), Chilopoda (Scolopendromorpha), Diplopoda, Gastropoda (Achatinidae), Insecta (Coleoptera, Hymenoptera, Isoptera, Orthoptera, insect larvae), and Amphibia (Ranidae). Although prey of caecilians are similar to those of other amphibians (such as in Anurans), the Goa caecilian Gegeneophis goaensis (family Indotyphlidae) has been found in the stomach of Ichthyophis cf. bombayensis (Venu et al. 2016). In a separate sample, Gurupur caecilian Gegeneophis krishni was found in the posterior part of the small intestine of Ichthyophis kodaguensis (Venu et al. 2016). These two examples are similar to our observation of Sylvirana mortenseni cannibalizing a juvenile (Figure 7B). Cannibalism has been reported for some amphibian species. For example, Pizzatto and Shine (2008) reported that the cane toad Rhinella marina (family Bufonidae) frequently consumed smaller metamorphs. Further, the cane toad exhibits cannibalism when seasonal precipitation increases encounter rates between large and small toads. Finally, Xenopus longipes (family Pipidae) was reported to have consumed the carcass of another conspecific (Doherty-Bone et al. 2018). 


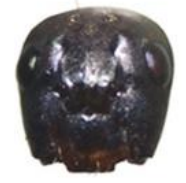

A

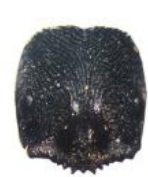

B

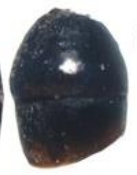

C

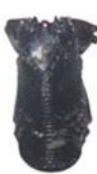

D

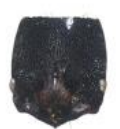

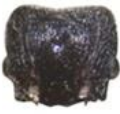

$\mathbf{E}$

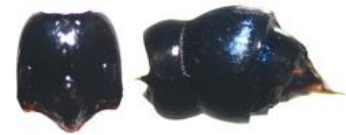

F $\quad$ G

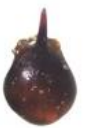

M

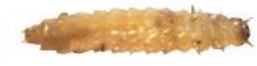

$\mathbf{N}$
H

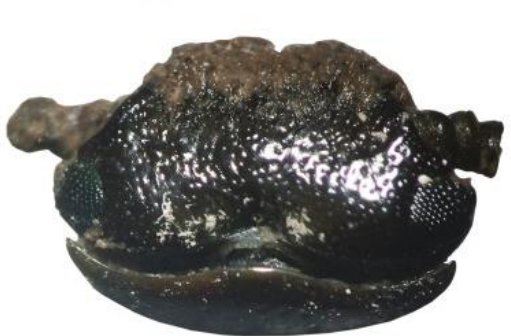

$\mathbf{O}$

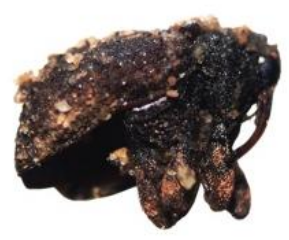

T

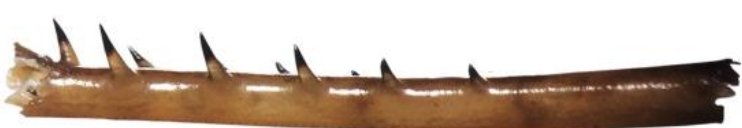

Ab

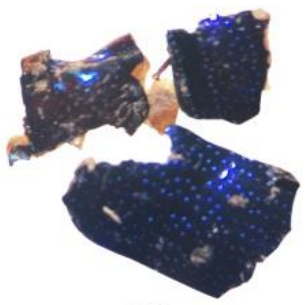

U

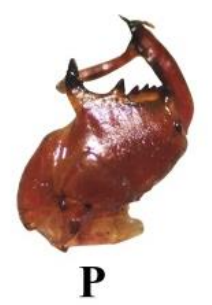

L

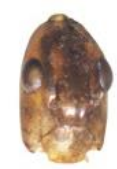

$\mathbf{Q}$

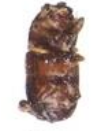

V

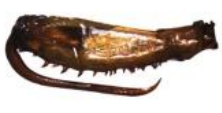

Y

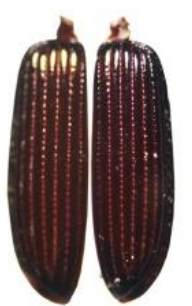

Ac

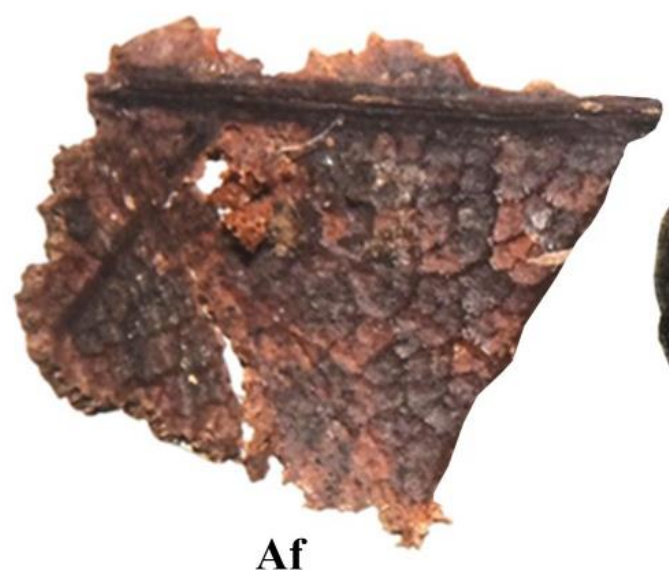

Ag

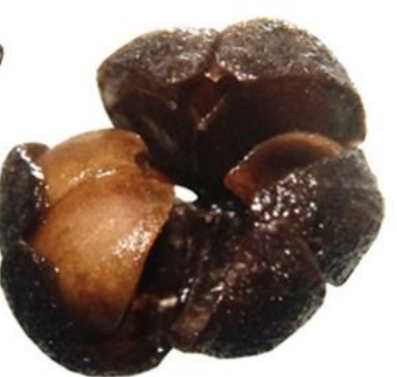

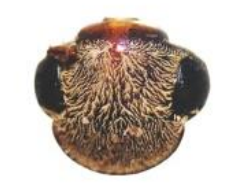

R

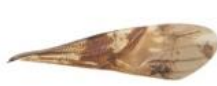

W

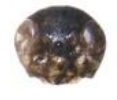

Z

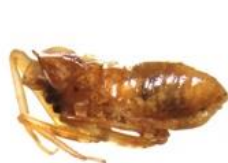

Ad

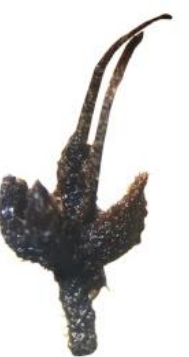

Ah

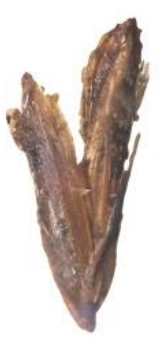

Ai

\section{$2 \mathrm{~mm}$}

Figure 8. Selected prey items found in amphibian fecal pellets collected from Yoddom Wildlife Sanctuary. A. Camponotus sp., B-D. Odontoponera denticulata, E. Pheidole sp., F-G. Leptogenys sp., H. Crematogaster aurita, I. Ectomomyrmex sp., J. Carebara diversa, K. Tetramorium sp., L. Calyptomyrmex rectopilosus, M. family Termitidae, N. order Coleoptera (larva), O. family Zephroniidae, P. family Hymenopodidae, Q. order Orthoptera, R. family Scarabaeidae, S. family Elateridae, T. family Curculionidae, U. family Chrysomelidae, V. family Scolytidae, W. order Hemiptera, X. family Cerambycidae, Y. family Reduviidae, Z. order Diptera, Aa. family Lucanidae, Ab. family Acrididae, Ac. family Tenebrionidae, Ad. class Arachnida, Ae. bryophyte (division Bryophyta), Af. dicot angiosperm leaf, Ag. dicot angiosperm fruit, Ah. dicot angiosperm flower, Ai. family Poaceae. 
An ongoing question remains as to how amphibians can occupy the same habitat and consume similar prey. In our study, four species of Microhyla (Microhyla fissipes, Microhyla butleri, Microhyla pulchra, Microhyla heymonsi) and a single species in genus Micryletta (Micryletta inornata) were found to occupy the same habitat and are normally observed in the same area under moist leaf litter. Fecal pellet analysis revealed that these species consume similar foods. Odontoponera denticulata was observed in the feces of Microhyla fissipes, Microhyla butleri, Microhyla pulchra and Microhyla heymonsi. Carebara diversa was found in the feces of Microhyla fissipes, Microhyla butleri, Microhyla heymonsi, and Micryletta inornata. In addition, other ant and termite species were observed in the feces of these microhylid frogs. Our results may suggest that their habitat provides an overabundance of prey, allowing these species to occupy the same area. Previous examples have suggested that the genus Odontoponera is the dominant ground-nesting ant in Thailand (Sitthicharoenchai and Chantarasawat 2006) and Vietnam (Eguchi et al. 2004). Furthermore, Odontoponera denticulata, which was found to be a prey item in our study, has been reported from the Philippines to throughout continental southeast Asia (Yamane et al. 2003; Eguchi et al. 2005; Jaitrong and Nabhitabhata 2005; Hannan 2007; Jaitrong et al. 2016).

Insects are the most diverse group of animals in the world (Sreedevi et al. 2015), and they were the dominant prey for amphibians in our study. Therefore, negative impacts to insect populations may affect amphibian populations in turn. Toxic compounds can accumulate in predators through consumption of contaminated prey, and vertebrates cannot excrete certain toxins found in prey items (Sánchez-Bayo 2012). Consequently, amphibians in agricultural areas which use pesticide may consume contaminated insects and accumulate toxins (Islam and Malik 2018). Although we could not identify all prey items consumed by amphibians, we provide insight into amphibian diet and ecology. Habitat inferences can be drawn from observations of amphibian prey; for example, we note that amphibians consuming Odontoponera denticulata (ground-nesting ant) can live in areas far from large water bodies. Combined with the results of other diet analyses, our prey item data are valuable for wildlife management in protected areas and may help inform future aquaculture practices.

There are advantages and drawbacks to the various methodologies available for studying amphibian diets. One such methodology is analysis of stomach contents, which is advantageous for identifying prey items as they are still partially intact (Buchler 1976), but this requires euthanasia of all target specimens (Kuzmin 2000). Another approach involves identifying prey fragments from the digestive tract. Stomach and gut contents are extracted from the digestive tract, sorted, counted, and classified to the lowest taxonomic category (Agudelo-Cantero et al. 2015). Perhaps the most widely used methodology to study prey items in living specimens is stomach flushing. Animals are physically manipulated to induce vomiting, and the stomach contents are analyzed (Kuzmin 2000). A variation of this protocol was used on 15 anuran species from an Araucaria forest in Brazil. The authors did not render the specimens unconscious for the procedure, as is normally done to reduce negative effects to the animal, but used a soft silicone infusion tube to prevent gut tissue lesions. Their results indicated that 29 individuals of the 583 specimens which were kept in captivity for one month, all of them survived and fed normally post-procedure (Solé et al. 2005). Another study examining the effects of stomach flushing focused on 143 specimens of rainbow lizard Agama agama (family Agamidae). The authors showed a lower survival rate among individuals that underwent stomach flushing compared with those that did not, for all sex and age classes. This suggests that stomach flushing increases mortality risk, and in addition, the results from these techniques are relatively similar to non-invasive fecal analysis (Luiselli et al. 2011). Due to the disadvantages discussed above associated with other prey item collection methods, we performed a fecal pellet analysis in our study. Chuaynkern et al. (2009) examined a pellet obtained from Kalophrynus interlineatus from southeastern Thailand and found it to contain a variety of insects. This finding was similar to that of Ponpituk et al. (2005), who collected 201 fecal pellets from Limnonectes gyldenstolpei, Limnonectes blythii, and Limnonectes taylori from various parts of Thailand, and found prey items belonging to 46 families in 16 orders. This technique is also used in reptiles (Thongproh et al. 2019). Belwood and Fenton (1976) gave a critique of a fecal analysis in a study focused on the little brown bat Myotis lucifugus (family Vespertilionidae). They note that the soft body parts of insects may be fully digested in fecal pellets, and thus some prey fragments cannot be identified, leading to errors in the estimation of quantity and type of insect prey consumed by animals. A similar pattern was expressed by Kunz and Whitaker (1983), where the authors note that whole or partial insects may be so digested to the extent of being unidentifiable. Analyzing prey items by different methodologies, such as stomach content or fecal pellet collection, and using different estimates, such as percent volume, percent frequency, or total enumeration, can lead to different findings and interpretations in amphibian studies.

\section{ACKNOWLEDGEMENTS}

We would like to express our thanks to Khon Kaen University (KKU), Kasetsart University (KU), the National Science Museum (NSM), and the Department of National Parks, Wildlife and Plant Conservation for their parts in facilitating our work. We also thank the Department of Biology (KKU), the Applied Taxonomic Research Center (KKU), and Yoddom Wildlife Sanctuary (Department of National Parks, Wildlife and Plant Conservation) for their funding support. This work is partially funded by the Research Fund for Supporting Lecturer to Admit High Potential Student to Study and Research on His Expert Program Year 2019 for Chantip Chuaynkern and 
Prapaiporn Thongproh. For assistance in the field, our special thanks go to Nithina Kaewtongkum, Pechrkawin Nurngsomsri, Benjawan Moonarsa, Teerawat Srisuk, Thanawhat Kaewboribut, Nattapon Surachotmongkol, and the staff of Yoddom Wildlife Sanctuary. We also thank Ruttapol Srisonchai and Kanyakorn Piraonapicha for their valuable expertise. Finally, we would like to thank the Department of National Parks, Wildlife and Plant Conservation for their permission to conduct this research in Yoddom Wildlife Sanctuary.

\section{REFERENCES}

Agudelo-Cantero GA, Castaño-Valencia RS, Castro-Herrera F, FierroPérez L, Asencio-Santofimio H. 2015. Diet of the blue-bellied poison frog Andinobates minutus (Anura: Dendrobatidae) in two populations from the Colombian Pacific. J Herpetol 49(3): 452-461.

Aowphol A, Rujirawan A, Taksintum W, Chuaynkern Y, Stuart BL. 2015. A new caruncle-bearing Limnonectes (Anura: Dicroglossidae) from northeastern Thailand. Zootaxa 3956(2): 258-270.

Belwood JJ, Fenton MB. 1976. Variation in the diet of Myotislucifugus (Chiroptera: Vespertilionidae). Can J Zool 54: 1674-1678.

Buchler ER. 1976. Prey selection by Myotis lucifugus (Chioptera: Vespertilionidae). Am Nat 110: 619-628.

Chan-ard T. 2003. A Photographic Guide of Amphibians in Thailand. Darnsutha Press Co., Ltd., Bangkok. [Thai]

Chuaynkern Y. 2005. Note on new distribution record of the Dalat frog Rana milleti Smith, 1921 in southeastern Thailand. Thailand Nat Hist Mus J 1: 189-190.

Chuaynkern Y, Chuaynkern C. 2012. Checklist of amphibians in Thailand. J Wildl Thailand 19(1): 163-211. [Thai]

Chuaynkern Y, Duengkae P. 2014. Decline of amphibians in Thailand. In: Heatwole H, Das I (eds) Conservation Biology of Amphibians of Asia. Status of Conservation and Decline of Amphibians: Eastern Hemisphere. Natural History Publications, Borneo.

Chuaynkern Y, Ohler A, Inthara C, Kumtong P, Dubois A. 2004. The recent distribution of Rana milleti Smith, 1921 in mainland Southeas Asia with the first record at Cambodia. Nat Hist J Chulalongkorn Univ 4(1): 1-13.

Chuaynkern Y, Wongwai A, Duengkae P, Hasin S. 2009. Kalophrynus interlineatus (Striped Sticky frog): Diet. Herpetol Rev 40(2): 205.

Department of National Park, Wildlife and Plant Conservation. 2006. Yoddom Wildlife Sanctuary. Available on: http://web3.dnp.go.th/wildlifenew/animConserveDepView.aspx?depI $\mathrm{d}=84$. [28 November 2017]

Department of National Park, Wildlife and Plant Conservation. 2012. Situation of illegal rosewood in Ubon Ratchathani Province. Available on: http://www.ubonratchathani.go.th/meeting 55/apr55/3.6.pdf. [6 June 2017]

Doherty-Bone TM, Nyingchia ON, Tapley B. 2018. Cannibalism in the critically endangered lake Oku clawed frog: a possible cause of morbidities and mortalities? Herpetol Notes 11: 667-669.

Duengkae P, Chuaynkern Y, Chaiyes A, Ponpituk Y, Siri S. 2016. Amphibians of Thailand. Biodiversity-based Economy Development Office, Bangkok. [Thai]

Eguchi K, Bui TV, Yamane S. 2004. A preliminary study on foraging distance and nesting sites of ants in Indo-Chinese lowland vegetation (Insect, Hymenoptera, Formicidae). Sociobiology 43(3): 445-457.

Eguchi K, Bui VT, Yamane S, Okido H, Ogata K. 2005. Ant fauna of Ba $\mathrm{Vi}$ and Tam Dao, North Vietnam (Insecta, Hymenoptera, Formicidae). Bull Inst Trop Agr, Kyushu Univ 27(2004): 77-98.

Frost DR. 2019. Amphibian species of the world: an online reference. Version 6.0. Available on: http://research.amnh.org/herpetology/amphibia/index.html. [7 June 2019].

Hannan M. 2007. Ants collected in Savar, Dhaka, Bangladesh in 2005. Asian Myrmecol 1: 101-104.

Hayes TB, Falso P, Gallipeau S, Stice M. 2010. The cause of global amphibian declines: a developmental endocrinologist's perspective. J Exp Biol 213: 921-933.
Heatwole H, Das I. 2014. Conservation Biology of Amphibians of Asia. Status of Conservation and Decline of Amphibians: Eastern Hemisphere. Natural History Publications, Borneo.

Heyer WR, Donnelly MA, McDiarmid RW, Foster MS. 1994. Measuring and Monitoring Biological Diversity: Standard Methods for Amphibians. Smithsonian Institution Press, Washington DC.

Hughes JB, Round PD, Woodruff DS. 2003. The Indochinese-Sundaic faunal transition at the Isthmus of Kra: an analysis of resident forest bird species distributions. J Biogeogr 30: 569-580.

Inthara C, Chuaynkern Y, Duengkae P, Grosjean S. 2009. The tadpole of Quasipaa fasciculispina (Inger, 1970) from southeastern Thailand, with the description of its buccal anatomy. Alytes 26(1-4): 86-96.

Islam A, Malik MF. 2018. Impact of pesticides on amphibians: a review. J Toxicol Anal 1(2): 3.

IUCN. 2019. IUCN Red List of Threatened Species. Version 2017.3. Available on: www.iucnredlist.org. [23 March 2019].

Jaitrong W, Guénard B, Economo EP, Buddahakala N, Yamane S. 2016. A checklist of known ant species of Laos (Hymenoptera: Formicidae). Asian Myrmecol 8: 17-48.

Jaitrong W, Nabhitabhata J. 2005. A list of known ant species of Thailand (Formicidae: Hymenoptera). Thailand Nat Hist J 1: 9-54.

Kunz TH, Whitaker JO. 1983. An evaluation of fecal analysis for determining food habits of insectivorous bats. Can J Zool 61: 13171321.

Kupfer A, Maraun M. 2003. Ichthyophis kohtaoensis: Diet. Herpetol Rev 34: 226.

Kuzmin SL. 2000. A simple method of food sampling in live amphibians. Zetschr f Feldherpetol 6: 230-233.

Leakey R, Lewin R. 1996. The Sixth Extinction: Biodiversity and its Survival. Weidenfeld and Nicolson, London.

Luiselli L, Akani GC, Ebere N, Pérez-Mellado V. 2011. Stomach flushing affects survival/emigration in wild lizards: a study case with rainbow lizards (Agama agama) in Nigeria. Amphib Reptil 32: 253-260.

May RM. 2002. The future of biological diversity in a crowded world. Curr Sci 82: 1325-1331.

Nabhitabhata J, Chan-ard T. 2005. Thailand Red Data: Mammals, Reptiles and Amphibians. Office of Natural Resources and Environmental Policy and Planning, Bangkok.

National Research Council. 1992. Conserving biodiversity: a research agenda for development agencies. The National Academies Press, Washington, DC.

Ngo BV, Hoang NT, Ngo CD. 2014. Diet of the Bannan caecilian Ichthyophis bannanica (Amphibia: Gymnophiona: Ichthyophiidae) in Makong Delta, Vietnam. J Herpetol 48: 506-513.

Norval G, Huang SC, Mao JJ, Goldberg SR, Yang YJ. 2014. Notes on the diets of five amphibian species from southern Taiwan. Alytes 30: 6977.

Office of Natural Resources and Environmental Policy and Planning. 2017. Thailand Red Data: Vertebrates. Ministry of Natural Resources and Environment, Bangkok.

Ohler A, Dubois A. 1999. The identity of Elachyglossa gyldenstolpei Andersson, 1916 (Amphibia, Ranidae), with comments on some aspects of statistical support to taxonomy. Zool Scr 23: 269-279.

Phimmachak S, Richards SJ, Sivongxay N, Seateun S, Chuaynkern Y, Makchai S, Som HE, Stuart BL. 2019. A new caruncle-bearing fanged frog (Limnonectes, Dicroglossidae) from Laos and Thailand. Zookeys 846: 133-155.

Phusaensri S, Kaewboribut T, Phummisutthigoon S, Kaewtongkum N, Youjaroen M, Tongpun P, Khongcharoensuk H, Nurngsomsri P, Chuaynkern C, Duengkae P, Chuaynkern Y, 2018. Theloderma petilum (Anura: Rhacophoridae): a new country record for Thailand. Alytes 36: 289-299.

Pizzatto L, Shine R. 2008. The behavioral ecology of cannibalism in cane toads (Bufo marinus). Behav Ecol Sociobiol 63: 123-133.

Ponpituk Y, Songchan R, Duengkae P, Hasin S, Chuaynkern Y. 2015. Diet of Limnonectes in Thailand. Proceedings of 53rd Kasetsart University Annual Conference: Science, Genetic Engineering, Architecture and Engineering, Agro-Industry, Natural Resources and Environment. Kasetsart University, Bangkok, 3-6 February 2015. [Thai]

Ponpituk Y, Tasen W, Duengkae P, Chuaynkern Y. 2014. Diet of the Taylor's stream frog (Limnonectes taylori) in hill evergreen forest at Huai Kok Ma Biosphere Reserve, Chiang Mai Province. J Wildl Thailand 21: 26-40. [Thai]

Sánchez-Bayo F. 2011. Impacts of agricultural pesticides on terrestrial ecosystems. In: Sánchez-Bayo F, van den Brink PJ, Mann RM (eds) 
Ecological Impacts of Toxic Chemicals. Betham Science Publisher Ltd, USA.

Sheridan JA, Stuart BL. 2018. Hidden species diversity in Sylvirana nigrovittata (Amphibia: Ranidae) highlights the importance of taxonomic revisions in biodiversity conservation. PLoS ONE 13: e0192766. htttps://doi.org/10.1371/journal.pone.0192766

Sitthicharoenchai D, Chantarasawat N. 2006. Ant species diversity in the establishing area for Advanced Technology Institute at Lai-Nan Subdistrict, Wiang Sa District, Nan Province, Thailand. Nat Hist J Chulalongkorn Univ 6(2): 67-74.

Solé M, Beckmann O, Pelz B, Kwet A, Engels W. 2005. Stomach-flushing for diet analysis in anurans: an improved protocol evaluated in a case study in Araucaria forests, southern Brazil. Stud Neotrop Fauna Environ 40(1): 23-28.

Sreedevi S, Meshram N, Shashank PR. 2015. Insect taxonomy-basics to barcoding. In: Chakravarthy AK (ed) New Horizons in Insect Science: Towards Sustainable Pest Management. Springer India, Bangalore, India.

Stuart SN, Hoffmann M, Chanson JS, Cox NA, Berridge RJ, Ramani P, Young BE (Eds.). 2008. Threatened amphibians of the world. Lynx Editions, Barcelona, IUCN, Gland and Conservation International, Arlington.

Taylor EH. 1962. The amphibian fauna of Thailand. Univ Kans Sci Bull 43: 265-599.

Taylor EH, Elbel RE. 1958. Contribution to the herpetology of Thailand. Univ Kans Sci Bull 38: 1033-1189.

The Government Gazette. 2003. Government Gazette, 120(74) (1 August 2003): 1 .
Thongproh P, Chuaynkern C, Duengake P, Ratree P, Khunkanha W, Phecharat E, Kingwongsa W, Chuaynkern Y. 2019. A study of food items of reptiles in Yoddom Wildlife Sanctuary, Ubon Ratchathani Province by using fecal pellet analysis. In: Marod D (ed) Proceedings of Thai Forest Ecological Research Network, T-FERN\#8. Khon Kaen University, 24-25 January 2019. [Thai]

Thongproh P, Youcharoen M, Chuaynkern Y, Chuaynkern C, Ratree P, Duengkae P, Phetcharat E, Maiprom W, Ungprombundith W, Wongpaiseart T. 2018. On the identities of Rhacophorus jarujini Matsui and Panha, 2006 and Rhacophorus orlovi Ziegler and Köhler, 2001(Amphibia, Anura, Rhacophoridae) from Thailand. Maejo Int J Sci Technol 12(1): 36-50.

Triplehorn CA, Johnson NF. 2005. Borror and DeLong's Introduction to the Study of Insects. 7 Edition. Thompson Brooks/Cole, Belmont, California.

Venu G, Parwar SK, Venkatachalaiah G. 2016. An unusual diet of Ichthyophis caecilians (Amphibia: Gymnophiona). Curr Sci 111(5): 793-795.

White RE. 1983. A Field Guide to the Beetles. Houghton Miffin Company, New York.

Whittaker K, Koo MS, Wake DB. Vredenburg VT. 2013. Global declines of amphibians. In: Levin SA (ed.) Encyclopedia of Biodiversity. Second Edition, Volume 3. Academic Press, Waltham, MA.

Wild Animal Reservation and Protection Act. 1992. The Royal Thai Government Gazette, Volume 109, Part 15.

Yamane S, Bui VT, Ogata K, Okido H, Eguchi K. 2003. Ant fauna of Cuc Phuong National Park, North Vietnam (Hymenoptera: Formicidae). Bull Inst Trop Agr, Kyushu Univ 27: 51-62. 